\title{
Publisher Correction: Co-opted transposons help perpetuate conserved higher-order chromosomal structures
}

Mayank N. K. Choudhary, Ryan Z. Friedman, Julia T. Wang, Hyo Sik Jang, Xiaoyu Zhuo and Ting Wang*

\section{Publisher Correction to: Genome Biol}

https://doi.org/10.1186/s13059-019-1916-8

Following publication of the original paper [1], an error was reported in the processing of Fig. 2. The correct Fig. 2 is supplied below and the original article [1] has been corrected. The publishers apologize for the error.

\section{Authors' information}

Twitter handles: Mayank Choudhary (@mayanknkc), Ryan Friedman (@rfriedman22),

Julia Wang (@juliaTJW), Ting Wang (@twang5).

Published online: 07 February 2020

\section{Reference}

1. Choudhary MN, Friedman RZ, Wang JT, et al. Co-opted transposons help perpetuate conserved higher-order chromosomal structures. Genome Biol. 2020;21:16 https://doi.org/10.1186/s13059-019-1916-8.

\section{Publisher's Note}

Springer Nature remains neutral with regard to jurisdictional claims in published maps and institutional affiliations. 


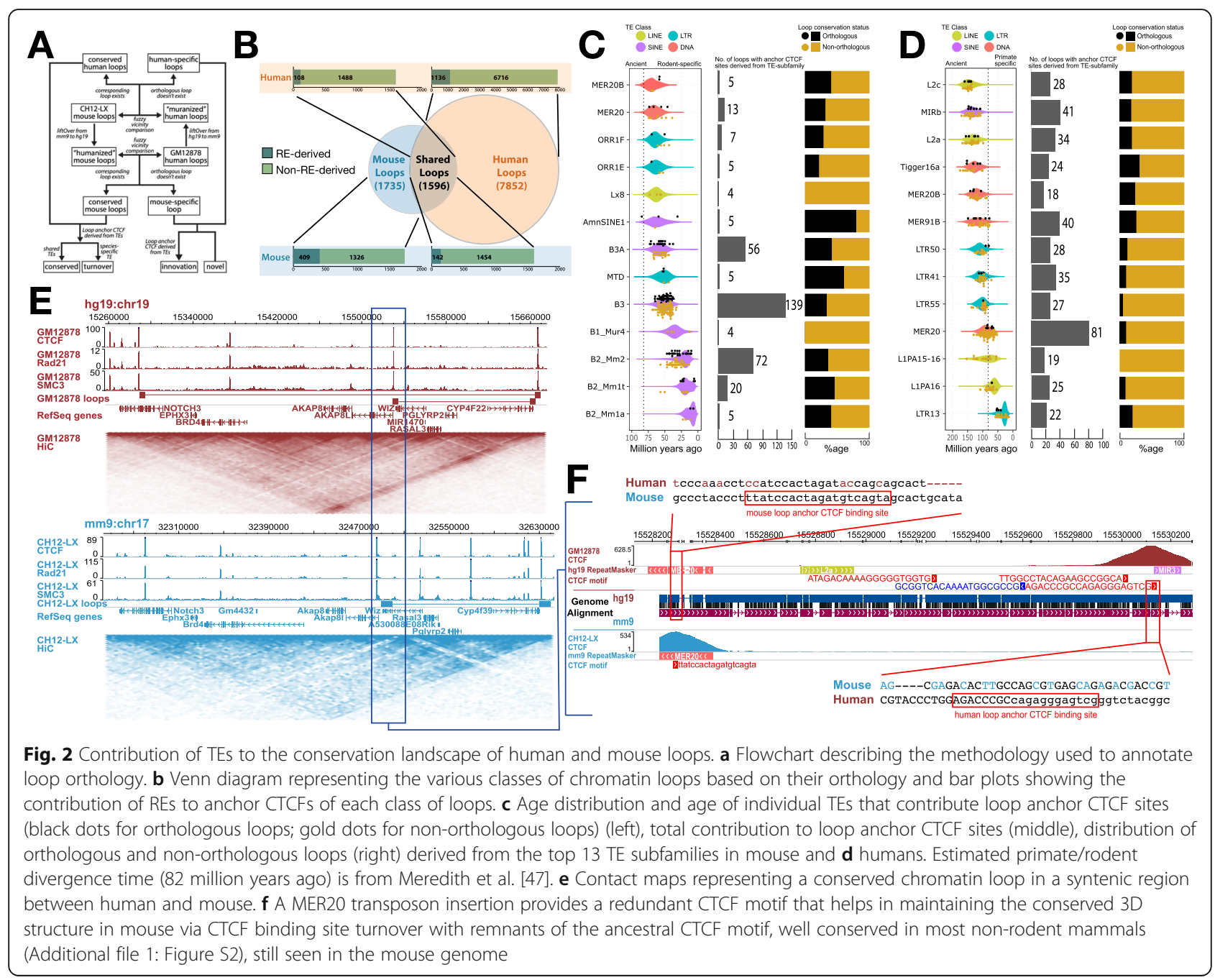

